arousal, relaxation, and autonomic tone. Headache 1990;30:145-51.

4 Sjaastad O. Cluster headache: on the inadequacy of existing hypotheses concerning the origin of the authomic Cephalalgia 1988;8:133-7.

5 Duke-Elder S. System of ophthalmology, vol XIII. London: Henry Kimpton, 1974:11.

6 Friedman AP. Headache. In: Baker AB, Joynt RJ, eds. Clinical neurology. Revised ed. Philadelphia: Harper and Row, 1985;2(13): $1-50$.

7 Hørven I, Russell D, Sjaastad O. Ocular blood flow changes in cluster headache and chronic paroxysmal hemicrania. Headache 1989;29: 373-6.

8 Gupta VK. Pupillary disturbances in migraine: what is the relation to autonomic dysfunction? $\mathcal{f}$ Neurol Neurosurg Psychiatry 1991;54: 847-8.

9 Drummond PD. Dissociation between pain and autonomic disturbances in cluster and autonomic disturbances in

10 Stewart DP, McIness A, Martin RWY, Fulton JD. Association of migrainous headaches with Gasserian ganglial
Med 1992;85:239-40.

\section{Peatfield replies:}

I wholly agree with Gupta that independent mechanisms for the headache and Horner's syndrome are required to explain the sequence of events in my patient. It is certainly possible the Horner's syndrome is entirely coincidental although the correlation in time suggests otherwise. Unfortunately, follow up information on my patient is not available.

I must confess to unwillingness to invoke a viral infection in parasympathetic ganglia to explain the pathogenesis of typical cluster headache, which even in this patient proved to be a spontaneously reversible periodic syndrome responding to corticosteroids among a wide variety of pharmacologically active agents.

Dr Gupta's second suggestion, that a purely mechanical ptosis can be related to a rise in intraocular pressure, is certainly ingenious but $I$ find it difficult to accept that a sudden rise in intraocular pressure sufficient to set up a trigeminal nerve mediated axon reflex would not first be painful.

R C PEATFIELD

The Princess Margaret Migraine Clinic,

Department of Neurology, Charing Cross Hospital,

Fulham Palace Road,
London W6 8RF, UK

\section{Non-invasive carotid imaging}

We agree with most of the comments made by Sellar in his comprehensive review of imaging techniques for displaying blood vessels of the head and neck. ${ }^{1}$ However, by contrast with Sellar, we would argue that conventional invasive angiography is no longer an essential component of the preoperative work up for prophylactic internal carotid endarterectomy; the combination of ultrasound and magnetic resonance angiography (MRA) is satisfactory for the vast majority of patients. In coming to this conclusion, we considered two key issues. Firstly, to what risk do we expose patients with symptomatic cerebrovascular disease when we perform invasive, intra-arterial contrast angiography? Secondly, do noninvasive techniques reliably identify appropriate patients? (using currently available ECST criteria this would be those patients with $\geqslant 70 \%$ stenosis of the symptomatic internal carotid artery).

In assessing the risk of angiography, Sellar "... suspect(s) that the complication rate remains at around $1 \%$ in most centres ...", but provides little appropriate prospective evidence to support this figure. The risks of conventional angiography clearly vary according to which group of patients are studied. Patients with symptomatic carotid stenosis seem to be at greater risk than other groups and several studies have shown that the risk increases with increasing degrees of stenosis. This is particularly important because, in many centres, patients with mild disease are effectively "screened out" by non-invasive techniques such as ultrasound. We therefore need to know the risks of angiography for the group of patients with moderate or severe stenosis, as these are the patients who will be exposed to the test. Few studies have looked specifically at this group. In a prospective study of 200 such patients in our centre, Davies and Humphrey found a $4 \%$ stroke rate in the 72 hours postangiography, with $2 \%$ death or serious disability. ${ }^{2}$ This figure intuitively seems high. However, it is not dissimilar to other studies, provided the appropriate patients are considered. In a study from Edinburgh, Hankey et al reported an overall stroke rate of $2 \%$ with a permanent deficit rate of $1 \cdot 3 \% .^{3}$ From their paper it is possible to calculate the postangiographic stroke rate in those patients with $\geqslant 50 \%$ stenosis. The figures are then an angiographic stroke risk of $5.5 \%$ and a permanent deficit risk of $3 \cdot 1 \%$. The benefits of carotid endarterectomy depend on a low surgical complication rate, as shown by the ECST and NASCET studies. Neither of these studies included the angiographic risk in their analyses. In borderline decisions, the risk of angiography, added to the surgical risk, could outweigh the benefits of endarterectomy.

The accuracy of non-invasive techniques is rightly assessed by comparison with invasive intra-arterial angiography, and Sellar quotes sensitivities for ultrasound of the order of $81 \%$ to $85 \%$. This is in agreement with a recent meta-analysis of non-invasive techniques which reported sensitivities of $83-86 \%$ and specificities of $89-94 \%$ for carotid ultrasound and MRA in detecting $70 \%$ stenosis or more. ${ }^{4}$ When interpreting sensitivity and specificity data it is important to realise that values less than $100 \%$ are inevitable, regardless of how accurate the method being assessed is. This is because of the variation in reporting that exists, both between different observers and also between the same observer reporting on separate occasions. Experienced neuroradiologists reporting the same conventional angiograms can disagree by considerable amounts. In a recent study we found that the mean magnitude of disagreement between consultant radiologists in our unit was $8-10 \%$ and that clinically important disagreements were reported in up to $6 \%$ of angiograms. ${ }^{5}$ Comparison of other modalities with angiography will inevitably result in sensitivities and specificities considerably less than $100 \%$, simply due to this variability in reporting.

Distinguishing occlusions from very tight stenoses can be reliably achieved with noninvasive tests. Colour duplex, in combination with continuous wave examination, has improved the ability of ultrasound to distinguish very tight stenoses from occlusions and, provided the correct $2 \mathrm{D}$ sequence is employed, MRA is highly reliable in making this distinction. Indeed, in situations of very slow flow, we have found that 2D MRA sequences can be easier to interpret than conventional angiograms.

As Sellar points out, ultrasound examination of the carotid arteries does require experienced operators if the results are to be relied on. This should not be used as an argument against the technique in general. By the same reasoning, we could argue for abandoning carotid endarterectomy itself, as the operation requires highly experienced surgeons, not available to all centres. The solution is to ensure a more thorough and effective training of ultrasonographers. We would strongly endorse the suggestion that prospective audit be maintained locally for any centre offering screening ultrasound and carotid endarterectomy.

The concern raised by Sellar regarding the signal gaps that appear on magnetic resonance angiograms beyond a tight stenosis has not proved to be a problem in our experience. ${ }^{6}$ Using 3D MRA techniques, we have found such signal gaps only in the presence of stenoses $>70 \%$ by ECST criteria. The paper quoting a signal gap associated with a $55 \%$ stenosis was a study using 2D MRA and, as suggested by Sellar, the stenosis was measured using NASCET criteria and so equivalent to a stenosis of $73 \%$ as measured by ECST criteria.

An argument often used in favour of continuing with invasive angiography is the issue of co-existent carotid siphon disease, which cannot be picked up reliably by duplex ultrasound. Our experience has been that significant siphon disease is a relatively uncommon finding. Furthermore, we are not convinced that its presence should alter the decision to perform endarterectomy, a similar conclusion to studies specifically looking at this question.?

In our unit we now use a combination of ultrasound and MRA for preoperative assessment of patients, and, like others, have found this highly successful. We reserve invasive angiography for those patients in whom the non-invasive investigations disagree significantly (an infrequent occurrence). In a situation where non-invasive methods clearly indicate the presence of a tight stenosis we do not think that it is justifiable to expose a patient to the risks of invasive angiography.

GR YOUNG

PRD HUMPHREY TE NIXON

The Walton Centre for Neurology and Neurosurgery NHS Trust, Rice Lane, Liverpool

Correspondence to: $\operatorname{Dr} G R$ Young.

1 Sellar RJ. Imaging blood vessels of the head and neck. $\mathcal{F}$ Neurol Neurosurg Psychiatry 1995;59:225-37.

2 Davies KN, Humphrey PR. Complications of cerebral angiography in patients with symptomatic carotid territory ischaemia screened by carotid ultrasound. $\mathcal{f}$ Neurol Neurosurg Py carotid ultrasound. $\mathcal{F}$.

3 Hankey GJ, Warlow CP, Molyneux AJ. Complications of cerebral angiography for patients with mild carotid territory ischaemia being considered for carotid endarterectomy. f Neurol Neurosurg Psychiatry 1990;53:542-8.

4 Blakeley DD, Oddone EZ, Hasselblad V Simel DL, Matchar DB. Noninvasive carotid artery testing: a meta-analytic review. Ann Intern Med 1995;122:360-7.

5 Young GR, Sandercock PAG, Slattery J, Humphrey PRD, Smith ETS, Brock L. Observer variation in the interpretation of intra-arterial angiograms and the risk of inappropriate decisions about carotid

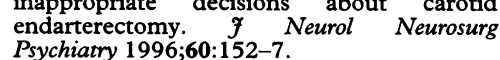

